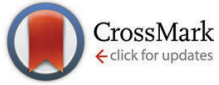

Cite this: Chem. Commun., 2014, 50, 11742

Received 17th June 2014, Accepted 7th August 2014

DOI: $10.1039 / \mathrm{c} 4 \mathrm{cc} 04594 \mathrm{~g}$

www.rsc.org/chemcomm

\section{Conformal sulfated zirconia monolayer catalysts for the one-pot synthesis of ethyl levulinate from glucose $\dagger$}

\author{
Gabriel Morales, ${ }^{a}$ Amin Osatiashtiani, ${ }^{b}$ Blanca Hernández, ${ }^{a}$ Jose Iglesias, ${ }^{a}$ \\ Juan A. Melero, ${ }^{* a}$ Marta Paniagua, ${ }^{a}$ D. Robert Brown, ${ }^{c}$ Marta Granollers, ${ }^{c}$ \\ Adam F. Lee ${ }^{\mathrm{b}}$ and Karen Wilson ${ }^{\star b}$
}

\begin{abstract}
Here we describe a simple route to creating conformal sulphated zirconia monolayers throughout an SBA-15 architecture that confers efficient acid-catalysed one-pot conversion of glucose to ethyl levulinate.
\end{abstract}

The US DoE has identified various platform chemicals obtainable via chemical or biochemical transformation of lignocellulosic biomass ${ }^{1,2}$ as renewable alternatives to existing fossil fuel derived chemicals. Key chemical intermediates that can be synthesised from biomass derived sugars include furanic components, such as 5-hydroxymethylfurfural (5-HMF) and furfural, ${ }^{3}$ and levulinic acid (LA) which offers a larger family of derivatives with significant commercial application (e.g. solvents, fuel additives and monomers). The latter's chemical versatility, reflecting the carboxylic acid and ketone functional groups, low cost and ready availability from 5 -carbon and 6-carbon sugars, suggests that LA could be a building block of central importance within future biorefineries. ${ }^{4}$ For example, alkyl levulinates have been proposed as excellent candidates for 'second generation' biofuels. ${ }^{5-8}$ Synthesis of such levulinates from cellulosic biomass has been previously reported, ${ }^{9-11}$ notably the acid catalysed esterification of LA with lower alkyl alcohols under severe reaction conditions utilising e.g. heteropolyacids, ${ }^{12,13}$ Amberlyst-15, acid zeolites and sulphated oxides; ${ }^{14}$ or sulfonic acid silicas, ${ }^{8}$ however such routes necessitate multistep processes and a source of high-purity LA (non-trivial to obtain due to contamination by polymeric humins). ${ }^{15}$

\footnotetext{
${ }^{a}$ Department of Chemical and Energy Tech, Chemical and Environmental Tech, Mechanical Tech and Analytical Chemistry, Universidad Rey Juan Carlos, C/Tulipan. s/n. E-28933, Móstoles, Madrid, Spain. E-mail: juan.melero@urjc.es; Fax: +34-91-488-74-68

${ }^{b}$ European Bioenergy Research Institute, Aston University, Birmingham B4 7ET, UK. E-mail: k.wilson@aston.ac.uk; Tel: +44 (0)121 2045456

${ }^{c}$ School of Applied Sciences, University of Huddersfield, Huddersfield, HD1 3DH, UK. E-mail: d.r.brown@hud.ac.uk; Tel: +44 (0)1484 473397

$\dagger$ Electronic supplementary information (ESI) available: Experimental details, porosimetry, XRD, TEM, XPS, ICP-OES, FTIR-pyridine, $\mathrm{NH}_{3}$-TPD and additional reaction data. See DOI: 10.1039/c4cc04594g
}

The one-pot production of alkyl levulinates directly from $\mathrm{C}_{6}$ sugars (Scheme 1) would thus provide a highly desirable alternative approach, especially attractive if abundant cellulosederived glucose could be reacted directly with an alcoholic medium, obviating the need for an LA feedstream. Use of an alcoholic medium would also help to minimise humin formation. ${ }^{15}$ Recent attempts to synthesize alkyl levulinates via homogeneous and heterogeneous acid catalysis ${ }^{16-21}$ suffer serious drawbacks including poor yields, high reaction temperatures which induce undesired dehydration of the alcohol solvent/reactant, and poor catalyst recyclability. ${ }^{22}$ Previous studies have also focused solely on the use of lower alcohols (methanol or ethanol), and not bulkier alcohols which enhance the properties of fuel end-products. Improvements in process efficiency for alkyl levulinate production require more active and selective solid acid catalysts. In particular, there is a need for bifunctional catalysts capable of directing the Lewis acid catalysed isomerisation of alkyl glucoside intermediates to alkyl fructosides, and their subsequent Brönsted acid catalysed dehydration to 5-alkoxymethylfurfural and esterification to form alkyl levulinate and formate (Scheme 1). The correct balance of Lewis and Brönsted acid sites is critical to the success of this complex tandem transformation.

We recently reported the preparation of bifunctional $\mathrm{SO}_{4} / \mathrm{ZrO}_{2}$ $(\mathrm{SZ})^{23}$ catalysts with tunable acid site distributions for 5-HMF production from glucose via a related reaction pathway, involving Lewis acid catalysed glucose isomerisation to fructose, followed by

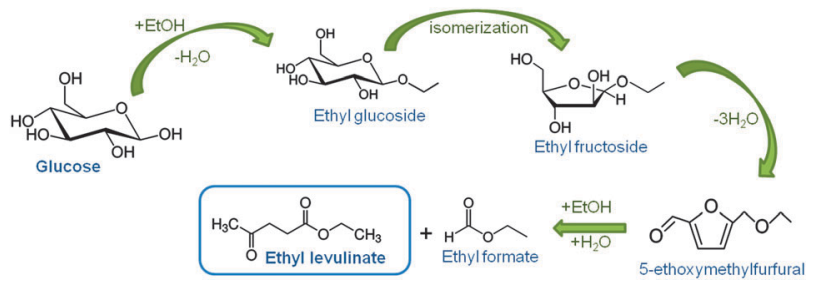

Scheme 1 Proposed reaction pathway for the acid-catalyzed conversion of glucose to ethyl levulinate in ethanol. ${ }^{16}$ 


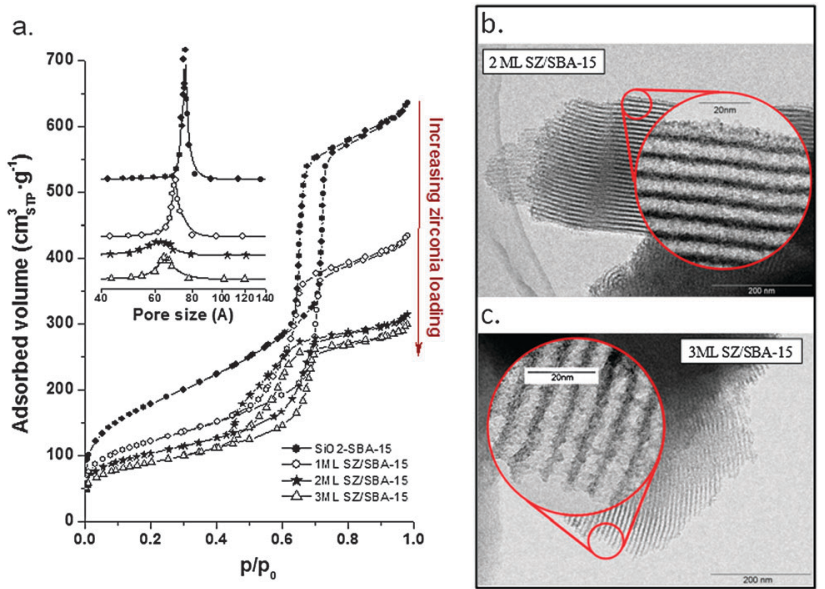

Fig. 1 (a) $N_{2}$ adsorption isotherms and pore size distributions; and (b, c) HRTEM of SBA-15 with grafted SZ layers.

Brönsted acid catalyzed dehydration to 5-HMF. The utility of such non-porous SZ catalysts is however limited by their inherently low surface areas (typically $<150 \mathrm{~m}^{2} \mathrm{~g}^{-1}$ ), and hence methodologies are required to stabilise either a highly porous or dispersed SZ phase. Here we report the preparation of high surface area, thermally robust SZ conformal monolayers with tunable Lewis:Brönsted acid site densities over a mesoporous SBA-15 template, for the tandem conversion of glucose to alkyl levulinates in alcoholic media.

Zirconia monolayers were first grown in a consecutive fashion over SBA-15, via sequential grafting and hydrolysis cycles employing a zirconium isopropoxide precursor. The uniformity of the subsequently sulfated grafted layers was confirmed by manometric porosimetry (Fig. 1a) which reveals a progressive decrease in pore volume, mean pore size and BET surface area with each grafting cycle, consistent with a layer-wise growth. Full analysis of the $\mathrm{N}_{2}$ adsorption-desorption isotherms via the $t$-plot method enables micro-, meso- and macropore volumes to be assessed and hence the influence of the grafting process on the textural properties of final materials. $t$-plot analysis (Tables S1 and $\mathrm{S} 2$, ESI $\dagger$ ) reveals a progressive decrease in pore volume on each grafting cycle, which is mainly associated with loss of mesoporosity and consistent with the preferential incorporation of $\mathrm{Zr}$ onto the surface of the mesoporous channels. This is supported by pore wall thickness calculations (Table S3, ESI $\dagger$ ). HRTEM (Fig. 1b and c and Fig. S1a and S2, ESI $\dagger$ ) confirms the long range order of the hexagonal SBA-15 phase is retained, and that the SBA-15 pore walls also remain uniform upon Zr grafting and sulfation, with no evidence for large crystallite deposition even after three grafting cycles.

XPS was employed to quantify the growth mode of the parent $\mathrm{ZrO}_{2}$ film, with attenuation of the Si $2 \mathrm{p}$ signal providing a direct measure of film thickness. Fig. 2a shows an exponential decay in the SBA-15 substrate intensity with consecutive $\mathrm{Zr}$ deposition, indicative of a layer-by-layer growth mode. The first and second grafting cycles attenuate the substrate by 35.6 and $51.9 \%$, equating to 0.5 and $0.84 \mathrm{~nm}$ thick adlayers of $\mathrm{ZrO}_{2}$ respectively. This is in excellent agreement with the thickness
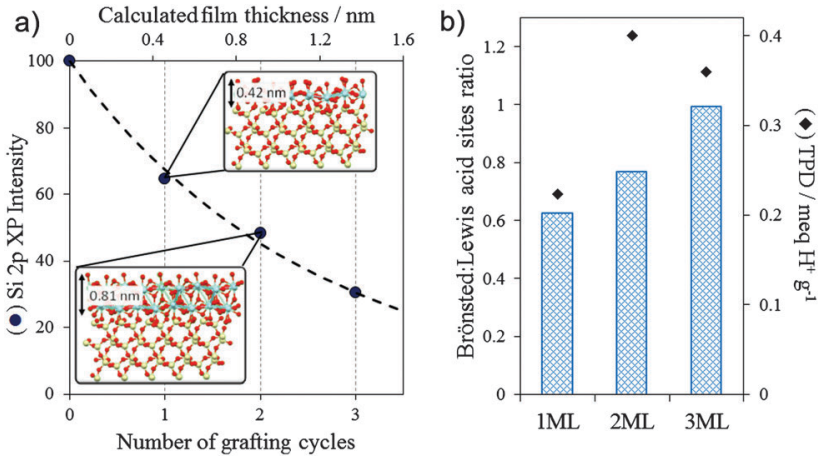

Fig. 2 (a) Si $2 p$ XP attenuation demonstrating layer-by-layer growth of $\sim 0.42 \mathrm{~nm}$ thick $\mathrm{ZrO}_{2}$ sheets. Dashed line shows the theoretical fit and associated film thickness to achieve the observed attenuation; (b) Brönste$\mathrm{d}$ :Lewis acid ratio from pyridine titration and $\mathrm{H}^{+}$density determined by TPD.

of a (111) oriented monolayer of monoclinic $\mathrm{ZrO}_{2}(0.42 \mathrm{~nm})$, confirming the successful growth of conformal $\mathrm{ZrO}_{2}$ monolayers over SBA-15.

The composition, morphology and surface acidity of these grafted $\mathrm{ZrO}_{2}$ layers upon sulfation with $0.075 \mathrm{M} \mathrm{H}_{2} \mathrm{SO}_{4}$ was further probed by $\mathrm{XRD}, \mathrm{XPS}, \mathrm{NH}_{3}$ calorimetry, and pyridine titration. The absence of wide angle XRD patterns (Fig. S1b, ESI $\dagger$ ) confirms that large SZ crystallites were not formed upon sulfation and calcination. Furthermore, the $\mathrm{Zr}$ :Si atomic ratios from XPS and EDX (Fig. S3, $\mathrm{ESI} \dagger$ ) remain unchanged by sulfation, suggesting there is minimal sintering of the $\mathrm{ZrO}_{2}$ film during impregnation with $\mathrm{H}_{2} \mathrm{SO}_{4}$ and re-calcination. Bulk and surface $\mathrm{Zr}$ and $\mathrm{S}$ contents for each sample (Table S4, ESI $\dagger$ ) show the $\mathrm{S}: \mathrm{Zr}$ ratio falls with $\mathrm{ZrO}_{2}$ thickness, consistent with surface sulfation.

Acid site strength and loading was probed by $\mathrm{NH}_{3}$ calorimetry (Fig. S5 and Table S5, ESI $\dagger$ ). The 1 and 2 ML samples exhibit a small proportion of sites at low $\mathrm{NH}_{3}$ coverage with $-\Delta H_{\text {ads }} \sim 180-200 \mathrm{~kJ} \mathrm{~mol}^{-1}$, attributable to strong Lewis acid sites. ${ }^{24}$ All grafted SZ/SBA-15 samples also exhibit sites of moderate acidity having a $-\Delta H_{\text {ads }} \sim 100-120 \mathrm{~kJ} \mathrm{~mol}^{-1}$, consistent with Brönsted acid sites, with the $2 \mathrm{ML}$ SZ/SBA-15 sample possessing the highest acid site loading. It is also interesting to note that pyridine titration (Fig. 2b and Fig. S7, ESI $\dagger$ ) shows that the Brönsted:Lewis acid site distribution increases with each $\mathrm{ZrO}_{2}$ grafting cycle, suggesting that the initial $\mathrm{ZrO}_{2}$ layers are more defective or electronically perturbed by the underlying $\mathrm{SiO}_{2}$ substrate. Fig. $2 \mathrm{~b}$ also shows the variation in acid site loading (derived from $\mathrm{NH}_{3} \mathrm{TPD}$ ) with $\mathrm{ZrO}_{2}$ film thickness, which exhibits a maximum of $0.40 \mathrm{mmol} \mathrm{g}^{-1}$ for the 2 ML sample. This suggests that a complete surface coverage of acid sites is achieved once the second layer is completed, and the third grafted layer does not further enhance acid properties. The requirement for a $\mathrm{ZrO}_{2}$ bilayer to optimise acid site loading may reflect different speciation of $\mathrm{Zr}$ on the SBA-15 support. Crystallization of $\mathrm{Zr}$ into a tetragonal structure is necessary to achieve superacidity, ${ }^{25}$ and might be expected to require a bilayer to achieve the correct atomic arrangement. Indeed the envelope for the $\mathrm{Zr} 3 \mathrm{~d}$ XP spectra (Fig. S4, ESI $\dagger$ ) shifts slightly to lower binding energy with increasing layer thickness, 


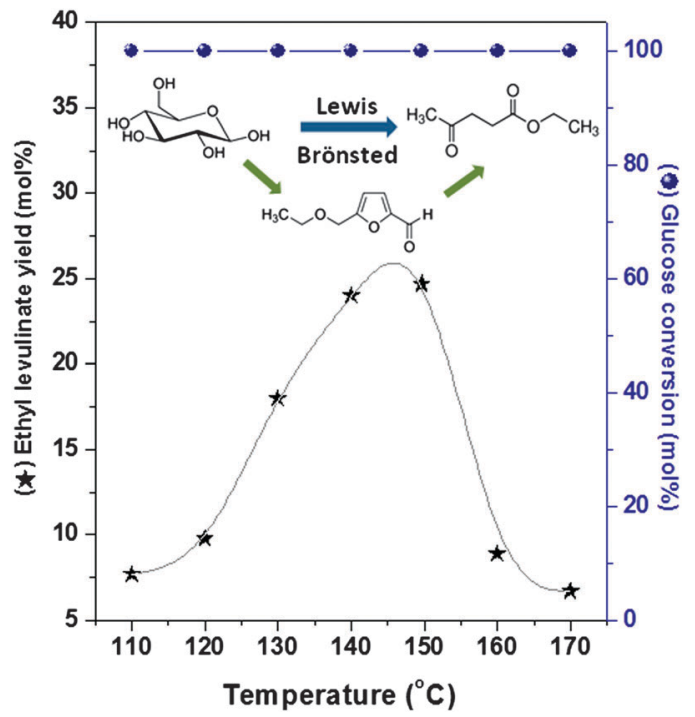

Fig. 3 Glucose conversion to ethyl levulinate as a function of reaction temperature over $2 \mathrm{ML}$ grafted SZ/SBA-15. Reaction conditions: $24 \mathrm{~h} ; 0.25 \mathrm{~g}$ of glucose; $2: 1$ glucose : catalyst mass ratio; $1: 100$ glucose : EtOH molar ratio.

consistent with electronic perturbation of the zirconia monolayer at the $\mathrm{SiO}_{2}$ interface.

The catalytic performance of the $2 \mathrm{ML}$ SZ/SBA-15, which possessed the optimum balance of Brönsted:Lewis acidity and acid site density, was subsequently evaluated for the direct production of ethyl levulinate (EL) from glucose (Fig. 3).

Complete glucose conversion was observed under all conditions, however the EL yield was sensitive to reaction temperature, displaying a volcano dependence with a maximum of $25 \mathrm{~mol} \%$ around $140-150{ }^{\circ} \mathrm{C}$. The fall in EL yield at high temperature coincided with the appearance of polymers from product degradation. Dispersing sulphated zirconia bilayers over a nanoporous SBA-15 template dramatically enhances the number (and uniformity) of accessible active sites, resulting in ethyl levulinate yields up to $141 \mathrm{~mol}_{\mathrm{EL}} \mathrm{mol}_{\mathrm{Zr}}{ }^{-1}$ (Fig. 4). This represents a significant improvement on the best literature performance, wherein amorphous bulk SZ, employed with poor $\mathrm{Zr}$ : glucose molar ratios of $\sim 0.7$, confer ethyl levulinate yields of only $44 \mathrm{~mol}_{\mathrm{EL}} \mathrm{mol}_{\mathrm{Zr}}{ }^{-1}$ at far higher temperatures $>200{ }^{\circ} \mathrm{C}$ which drive undesired intermolecular dehydration of ethanol to diethyl ether. ${ }^{16}$

Therefore, a clear advantage of our catalysts is the capability of reaching relatively high EL yields under moderate temperatures, while avoiding ethanol losses as diethyl ether. We attribute the enhanced low temperature activity of grafted SZ/SBA-15 to the presence of strong Lewis acid sites which drive glucose isomerization. Catalyst reusability was confirmed over three consecutive runs (Fig. S8, ESI $\dagger$ ), with intermediate calcination of the used catalyst at $550{ }^{\circ} \mathrm{C}$ to remove organic deposits, which revealed the yield to EL was maintained or even slightly increased. This evidences the stability of the sulfated $\mathrm{ZrO}_{2}$ monolayers grafted on SBA-15, and overcomes the extended leaching problems of commercial sulfated zirconias.

Preliminary experiments on the reactivity of fructose and HMF were also conducted to probe the rate-limiting step. The

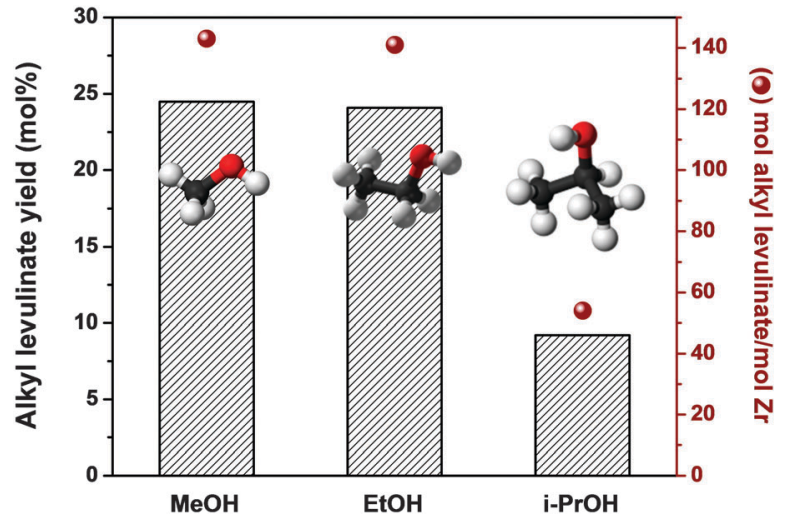

Fig. 4 Comparison of different alcohols for the conversion of glucose to alkyl levulinates over $2 \mathrm{ML}$ grafted SZ/SBA-15 solid acid catalyst. Reaction conditions: $140{ }^{\circ} \mathrm{C} ; 24 \mathrm{~h} ; 0.25 \mathrm{~g}$ of glucose; $2: 1$ glucose : catalyst mass ratio; $1: 100$ glucose: $\mathrm{ROH}$ molar ratio.

EL yield increased slightly using fructose instead of glucose (Table S6, ESI $\dagger$ ), indicating that ethyl glucoside isomerisation to ethyl fructoside occurs more slowly than the subsequent dehydration of ethyl fructoside to 5-ethoxymethylfurfural. In contrast, HMF conversion to EL was comparatively poor at $140{ }^{\circ} \mathrm{C}$, reflecting its instability with respect to humins, but conferred EL yields four times greater achieved with glucose at $130{ }^{\circ} \mathrm{C}$ (Fig. S9, ESI $\dagger$ ). A $\mathrm{ZrO}_{2}$ co-catalyst enhanced this slow isomerisation step and net EL yield (Table S7, ESI $\dagger$ ).

The versatility of grafted SZ monolayers towards alkyl levulinate production was further explored using methanol and isopropanol (Fig. 4). Methanol conferred a similar yield to ethanol, whereas isopropanol was less reactive, presumably a result of the bulkier intermediates and products in the production of isopropyl-levulinate. Since there are no previous reports on the telescopic production of isopropyl-levulinate from glucose, this represents an exciting development in the catalytic production of more complex alkyl-levulinates which would find wide application as plasticizing agents, solvents, and speciality chemicals.

Conformal SZ monolayers with tuneable surface acid strength and site density can be dispersed over a mesoporous SBA-15 framework through a simple wet chemical grafting/ hydrolysis protocol. A bilayer SZ/SBA-15 material exhibits the maximum surface acidity and balance of Lewis:Brönsted sites, and exhibits good performance in the one-pot conversion of glucose to alkyl levulinates under mild conditions.

Financial support from the Spanish Ministry of Economy and Competitiveness through the project CTQ2011-28216C02-01 is kindly acknowledged. KW thanks the Royal Society for an industry fellowship and the EPSRC for funding (EP/ K000616/2 and EP/K014676/1). AFL thanks the EPSRC for a Leadership Fellowship (EP/G007594/4).

\section{Notes and references}

1 T. Werpy and G. Petersen, Top Value Added Chemicals from Biomass, U. S. D. o. Energy, 2004.

2 J. J. Bozell and G. R. Petersen, Green Chem., 2010, 12, 539-554.

3 R.-J. van Putten, J. C. van der Waal, E. de Jong, C. B. Rasrendra, H. J. Heeres and J. G. de Vries, Chem. Rev., 2013, 113, 1499-1597. 
4 G. Centi, P. Lanzafame and S. Perathoner, Catal. Today, 2011, 167, $14-30$.

5 H. Joshi, B. R. Moser, J. Toler, W. F. Smith and T. Walker, Biomass Bioenergy, 2011, 35, 3262-3266.

6 E. Christensen, A. Williams, S. Paul, S. Burton and R. L. McCormick, Energy Fuels, 2011, 25, 5422-5428.

7 B. C. Windom, T. M. Lovestead, M. Mascal, E. B. Nikitin and T. J. Bruno, Energy Fuels, 2011, 25, 1878-1890.

8 J. A. Melero, G. Morales, J. Iglesias, M. Paniagua, B. Hernandez and S. Penedo, Appl. Catal., A, 2013, 466, 116-122.

9 M. Mascal and E. B. Nikitin, ChemSusChem, 2010, 3, 1349-1351.

$10 \mathrm{~J}$. P. Lange, E. van der Heide, J. van Buijtenen and R. Price, ChemSusChem, 2012, 5, 150-166.

11 S. Dutta, S. De, B. Saha and M. I. Alam, Catal. Sci. Technol., 2012, 2, 2025-2036.

12 G. Pasquale, P. Vazquez, G. Romanelli and G. Baronetti, Catal. Commun., 2012, 18, 115-120.

13 K. Yan, G. S. Wu, J. L. Wen and A. C. Chen, Catal. Commun., 2013, 34, 58-63.

14 D. R. Fernandes, A. S. Rocha, E. F. Mai, C. J. A. Mota and V. T. da Silva, Appl. Catal., A, 2012, 425, 199-204.
15 X. Hu, C. Lievens, A. Larcher and C. Z. Li, Bioresour. Technol., 2011, 102, 10104-10113.

16 L. C. Peng, L. Lin, J. H. Zhang, J. B. Shi and S. J. Liu, Appl. Catal., A, 2011, 397, 259-265.

17 M. Watanabe, Y. Aizawa, T. Iida, T. M. Aida, C. Levy, K. Sue and H. Inomata, Carbohydr. Res., 2005, 340, 1925-1930.

18 K.-i. Tominaga, A. Mori, Y. Fukushima, S. Shimada and K. Sato, Green Chem., 2011, 13, 810-812.

19 S. Saravanamurugan and A. Riisager, Catal. Commun., 2012, 17, 71-75.

20 C. Chang, G. Xu and X. Jiang, Bioresour. Technol., 2012, 121, 93-99.

21 R. L. V. Mao, Q. Zhao, G. Dima and D. Petraccone, Catal. Lett., 2011, 141, 271-276.

22 M. Mascal and E. B. Nikitin, Green Chem., 2010, 12, 370-373.

23 A. Osatiashtiani, A. F. Lee, D. R. Brown, J. A. Melero, G. Morales and K. Wilson, Catal. Sci. Technol., 2014, 4, 333-342.

24 V. Dondur, V. Rakic, L. Damjanovic and A. Auroux, J. Serb. Chem. Soc., 2005, 70, 457-474.

25 C. Morterra, G. Cerrato and M. Signoretto, Catal. Lett., 1996, 41, 101-109. 